\title{
PROFESORES DE ENSEÑANZA MEDIA TÉCNICA AGRÍCOLA: MECANISMOS PARA LA APROPIACIÓN DEL CONOCIMIENTO CIENTÍFICO ${ }^{1}$
}

\author{
Pedro Gabriel Marcano Molano² \\ Gabriel de Araújo Santos ${ }^{3}$
}

\begin{abstract}
RESUMEN
En la presente investigación se exponen elementos teóricos actualizados sobre los procesos de transferencia de ciencia, tecnología e innovación utilizados por profesores de Colegios técnicos agrícolas en el contexto latinoamericano. En la actualidad la mayoría de artículos científicos se vuelcan a la tarea de explicar el fenómeno de transferencia de tecnología desde la perspectiva empresarial existiendo un déficit de información sobre este proceso en otras esferas del desarrollo socioeconómico de los países de la región. El objetivo del presente trabajo determinar la relación existente entre el conocimiento científico, mecanismos para la de transferencia de tecnología y apropiación de conocimientos en profesores de enseñanza media técnico agrícola a partir de la metodología de revisión y análisis documental (RAD) con artículos científicos de las bases de datos Gale Cengage Learning, Academic One File y Ebscot. Entre los resultados más relevantes encontramos que los mecanismos de transferencia utilizados con mayor frecuencia por los profesores son las publicaciones científicas en formato digital, pero no en bases indexadas; destacan además los "spin-offs" académicos, licencias y patentes, se evidenció la importancia de las nuevas tecnologías de la comunicación.
\end{abstract}

Palabras clave: transferencia de tecnología, apropiación de conocimiento, técnico agrícola; formación continua.

\section{PROFESSORES DO ENSINO MÉDIO TÉCNICO AGRÍCOLA: MECANISMOS PARA A APROPRIAÇÃO DO CONHECIMENTO CIENTÍFICO}

\section{RESUMO}

Nesta pesquisa são apresentados os fundamentos teóricos atualizados sobre o processo de transferência da ciência, tecnologia e inovação utilizadas pelos professores de ensino médio técnico agrícola na América Latina. Atualmente, a maioria dos artigos científicos volta-se para a tarefa de explicar o fenômeno da

1 Institución Proveedora de recursos para la investigación: Coordenação de aperfeiçoamento de pessoal de nivel superior (CAPES).

2 Graduado en Psicología (UH - Cuba). Maestría en Educación (UFRRJ). Profesor de la Universidad Estatal Península de Santa Elena, Ecuador. E-mail: pmarcano@upse.edu.ec

3 Graduado en Agronomía (UFRRJ). Maestría en Agronomía (UFRRJ). Doctorado en Química del Suelo (École Nationale Superieure d'Agronomie e des Industries Alimentares de Nancy, Francia). Posdoctorado en Educación Agrícola (Ecole Nationale de Formartion Agronomique de Toulouse, Francia). Profesor de la Universidad Federal Rural de Rio de Janeiro. E-mail: gasantos@ufrrj.br 
transferência de tecnologia a partir da perspectiva empresarial, havendo uma falta de informação sobre esse processo em outros domínios do desenvolvimento socioeconômico dos países da região. O objetivo deste trabalho foi determinar a relação entre as variáveis "conhecimento científico" "mecanismos de transferência de tecnologia", "apropriação de conhecimentos" e "professores do ensino médio técnico agrícola" utilizando a metodologia de revisão e análise documental em artigos científicos das bases de dados Gale Cengage Learning, Academic One File e Ebscot Host. Entre os resultados mais relevantes, constatamos que os mecanismos de transferência utilizados com maior frequência pelos professores são as publicações científicas em formato digital, mas não de bancos de dados indexados; além disso, destacam-se os spin-offs acadêmicos, licenças e patentes. Foi evidenciada a importância das novas tecnologias de comunicação na transferência do conhecimento científico.

Palavras-chave: transferência de tecnologia, apropriação do conhecimento, ensino médio agrícola, formação continuada.

\section{INTRODUCCIÓN}

Con esta investigación se pretende dar a conocer elementos teóricos actualizados en torno a los mecanismos de transferencia de tecnología, ciencia, información e innovación utilizados por docentes de enseñanza media técnica agrícola. La presente investigación tiene relevancia, utilidad práctica, teórica y metodológica ya que los datos obtenidos sobre la apropiación de conocimientos científicos y actualización de contenidos por parte de los profesores de enseñanza media (formación continua) contribuirán a conocer mejor el fenómeno y contribuir en mejorar procesos a partir de la detección de barreras para la apropiación del conocimiento científico.

La bibliografía consultada resalta tres modelos generales de transferencia utilizados por los centros de producción científica para diseminar hallazgos, experiencias, productos y servicios. Estos modelos son el lineal, el dinámico y el de tres hélices, sin embargo; cada País, Universidad o centro de producción científica no adopta e institucionaliza un modelo literalmente, sino que lo adecúa en dependencia de su experiencia y sus necesidades. (GÓMEZ, MIRA y VERDÚ, 2007; COSTA y TORKOMIAN, 2008)

Se abordarán, en esta investigación, los elementos más importantes del proceso de apropiación de conocimientos por parte de los profesores y su relación con la transferencia de ciencia y tecnología desde los centros de producción científica, es decir; los mecanismos por los cuales se llevan a cabo, estos pueden ser proyectos de investigación, cursos de formación y actualización conjuntos entre centros de investigación y los de Enseñanza Media, desarrollo de tesis de Maestría y Doctorales entre otros. La revisión teórica se realiza a partir de las relaciones lógicas entre nuestras variables de estudio presentes en artículos científicos de los últimos diez años con un formato metodológico de recolección de datos y análisis de resultados acorde al ámbito académico.

El objetivo del presente trabajo fue determinar cómo se produce la apropiación de conocimientos científicos en profesores de Colegios técnicos agrícola y su relación con los mecanismos para la transferencia de tecnología mediante la metodología de revisión y análisis documental (RAD). Se espera contribuir con correlaciones de variables que contribuyan en el análisis teórico de cómo se realiza el proceso de apropiación de conocimientos y la relación con la transferencia de 
ciencia, tecnología e innovación utilizados por los centros de producción científica en Latinoamérica.

\section{REFERENCIAL TEÓRICO}

A continuación se detallan algunos conceptos que constituyen el punto de partida de la presente investigación, el primero de ellos es la noción de transferencia, Souza (1983) considera que para que exista el proceso de transferencia de tecnología o información es necesario que exista un desplazamiento de información, por lo general constituye un conjunto de información en términos de conocimientos y prácticas tecnológicas de un lugar a otro. El proceso es efectivo una vez que al producirse la transferencia de tecnología, ésta se absorbe y permite al receptor utilizar el conocimiento para innovar.

Se podría definir la transferencia de tecnología aplicada al ámbito docente como un proceso que va más allá de la venta de productos y servicios comercializados por beneficios socio-económicos. Según Flores (2009) son las relaciones que hacen los profesores en el espacio y tiempo de la formación produciendo generalizaciones en las que se manifiestan las competencias y concepciones de los docentes re-actualizándose en la práctica a través de las acciones. La transferencia de tecnología, ciencia e innovación vista desde la perspectiva de los docentes se resume en los descubrimientos y resultados de procesos investigativos de forma general y la búsqueda de aplicaciones prácticas que serían útiles para la humanidad (FLORES, G. 2003; FLORES, G. 2009; ALESSANDRINI, A., 2013).

El Conocimiento Científico es otra categoría que necesita ser definida teóricamente, según Marconi y Lakatos (2005) existen cuatro tipos de conocimiento, el popular, el filosófico, el religioso y el científico. El pensamiento científico tiene las siguientes características: real, contingente, sistemático, verificable, falible y aproximadamente exacto. De acuerdo con Moraes et al (2011) este tipo de pensamiento es real ya que trata de sucesos o hechos; es contingente porque sus preposiciones o hipótesis tienen su veracidad conocida a través de la experiencia; es sistemático porque se trata de un conocimiento lógicamente ordenado, formando un sistema de ideas y no conocimiento desconectado; verificable ya que las afirmaciones no verificables no pertenecen al ámbito de la ciencia; es falible puesto que no es definitivo, absoluto o final; y es aproximadamente exacto, es decir que se puede remodelar el cuerpo teórico existente debido a nuevas propuestas y desarrollo.

\footnotetext{
Los conocimientos científicos constituyen el resultado de la actividad científica investigativa. En concordancia con el materialismo dialéctico, el conocimiento es un producto de la actividad social laboral e intelectual que representa la reproducción ideal en forma lingüística de los nexos objetivos, regulares y prácticos del mundo objetivo transformado (GONZALEZ, 2006, p.5).
}

En ese mismo sentido, González (2006) señala que el conocimiento científico es la representación de la variedad de los fenómenos del mundo y de su esencia y se distingue, porque es indispensable que se exprese en forma lingüística y no sensorial -sensaciones, percepciones y representaciones-.

Resulta igualmente necesario definir los mecanismos de transferencia de conocimiento científico relatados con mayor frecuencia en la literatura especializada, 
según Costa y Torkomian (2008) los mecanismos de transferencia de tecnología son las vías que utilizan los centros de producción científica para diseminar sus hallazgos. Se describen a continuación tres de los mecanismos más utilizados en la actualidad y más relatados en la literatura.

En primer lugar tenemos las publicaciones científicas, autores como Russel (2000), Oppenheim, Greenhalgh y Rowland (2000) afirman que las publicaciones son fundamentales para el campo de la comunicación formal de la ciencia.

\begin{abstract}
La actividad científica está íntimamente relacionada con la comunicación de los resultados que se derivan de su práctica, lo que a través de la publicación científica tiene su máxima expresión. Si se considera que la ciencia es un fenómeno social, al igual que ocurre con otros sectores de la vida social, las actividades de investigación y de publicación son sensibles a los fenómenos socioeconómicos que tienen lugar en el entorno donde se desarrollan y su divulgación se constituye en evidencias del desarrollo científico (GONZÁLEZ et al, 2015, p.2).
\end{abstract}

En la actualidad, muchos expertos creen que las publicaciones académicas, de las cuales las dedicadas a la investigación son el principal vehículo, se encuentran actualmente en crisis ante la aparición de nuevas estructuras de comunicación. Con la aparición del internet y la facilidad para acceder a la información; desde los años noventa el internet se convirtió en el principal método para informar sobre nuevos hallazgos para los investigadores (GINSPARG P., 1996; RUSSEL 2000).

En segundo lugar se hace referencia a los Spin-Off académicos, en ese sentido, Daim y Lavoie (2007), refieren que se entiende como spin-off las iniciativas privadas en donde los directivos están vinculados a la universidad (profesores, investigadores, personal técnico o administrativo), o bien se crea en base al conocimiento o tecnología creada y propiedad de la institución.

Así mismo, Gómez, Mira y Verdú (2007) consideran que una de las vías de transferencia que más impulso ha tenido en la última década en las universidades a nivel internacional ha sido la creación de spin-offs académicas. Esta realidad ha estimulado el debate académico y político sobre la asociación entre este fenómeno y los mecanismos de contribución a la creación de riqueza (FELDMAN et al 2002)

Las empresas basadas en conocimiento son fundamentales para la revitalización de muchas regiones, especialmente aquellas que han experimentado un declive en las industrias tradicionales, mediante la generación de un amplio abanico de efectos positivos en el desarrollo económico (GÓMEZ, MIRA y VERDÚ 2007).

La transferencia de tecnología desde las universidades al mercado ha estado históricamente dominada por las licencias o Spin-off (COTEC, 2002).

Finalmente, el mecanismo de transferencia de conocimiento científico más relatado en las fuentes es el uso de Patentes, estas pueden ser definidas como un título que otorga el poder público al inventor por el cual se concede a este último el derecho a explotar, es decir que tiene derechos de comercialización una vez que los detalles de la invención se hacen públicos mediante su inscripción al registro de Patentes (COTEC, 2002; AUDRETSCH et al, 2006; NILSSON, RICKNE y BENGTSSON, 2010). 


\section{ASPECTOS METODOLÓGICOS}

El proceso de investigación utilizado para la recolección de datos y análisis de resultados fue la metodología documental en base a revisión bibliográfica descriptiva; el alcance de la investigación fue exploratorio aproximándonos a un estado del arte en torno al proceso de apropiación de ciencia, tecnología e innovación por parte de profesores de enseñanza media y los procesos de transferencia de ciencia y tecnología utilizados por los centros de producción científica para diseminar sus hallazgos.

Para la localización de los documentos bibliográficos se utilizaron varias fuentes, se realizó una búsqueda bibliográfica de documentos en inglés, español y portugués en el último trimestre del 2016 en las bases de datos Gale Cengage Learning, Academic One File y Ebscot Host utilizando los descriptores: mecanismos de transferencia de tecnología, apropiación de conocimientos y profesores de enseñanza media técnico agrícola. Se realizó además una búsqueda en internet utilizando "Google Académico" y repositorios de Universidades latinoamericanas con los mismos términos.

El enfoque metodológico empleado para sistematizar y analizar los resultados fue la revisión y análisis documental (RAD), a partir de la adaptación de los procedimientos metodológicos de Barbosa et al (2013). La sistematización de los descriptores encontrados se desarrolló a partir de la adaptación y aplicación de las fases de desarrollo de la metodología de revisión y análisis documental: heurística y hermenéutica. Se describen a continuación ambas fases utilizadas para la realización de esta investigación:

a) Fase heurística

En la fase heurística, se desarrolló un protocolo de búsqueda y revisión de fuentes de información y autores considerando los siguientes elementos: I) el idioma de las fuentes de información; II) antigüedad de la publicación; III) recursos de Información; IV) normas de revisión; V) criterios de Exclusión y VI) criterios de inclusión. Se sistematizó toda la información recolectada en fichas de revisión documental (ver cuadro 1). El cruce de variables y categorías de análisis plasmadas en las fichas de esta fase fueron organizadas según los criterios de inclusión para posteriormente ser sistematizados cualitativamente en la siguiente fase, analizándolas como un "todo". Se detalla a continuación en el Cuadro 1 la guía de recolección de datos utilizada en la fase heurística.

Cuadro 1 - Guía de recolección de datos de la fase heurística

protocolo de búsqueda de fuentes de información

idiomas: español, inglés y portugués

período de tiempo: $2004-2016$

Recursos de información (bases de datos):

Gale Cengage Learning.

Academic One File.

Ebscot Host.

Google Académico.

\begin{tabular}{l|l} 
normas de & Diagnosticar la existencia de trabajos análogos o cercanos sobre el \\
revisión & abjoto
\end{tabular} objeto de estudio y revisión de las fuentes de información teniendo 


\begin{tabular}{|l|l|}
\hline & $\begin{array}{l}\text { al horizonte de investigación (problema y objetivos) como referente } \\
\text { constante de revisión. } \\
\text { Corroborar la correspondencia de la fecha de publicación de la } \\
\text { fuente primaria y hacer lectura del resumen e introducción como } \\
\text { estrategia de inclusión o exclusión preliminar. }\end{array}$ \\
\hline $\begin{array}{l}\text { criterios de } \\
\text { exclusión }\end{array}$ & $\begin{array}{l}\text { Publicaciones que no contengan: información de interés, contenido } \\
\text { educativo y diseños investigativos no se correspondan con el } \\
\text { contexto académico. }\end{array}$ \\
\hline $\begin{array}{l}\text { criterios de } \\
\text { inclusión }\end{array}$ & $\begin{array}{l}\text { Publicaciones que contengan: relaciones lógicas entre nuestras } \\
\text { variables de estudio, formato metodológico de recolección de datos } \\
\text { y análisis de resultados acorde al ámbito académico. }\end{array}$ \\
\hline
\end{tabular}

Fuente: Basado en Barbosa, J. et al (2013)

b) Fase hermenéutica

El análisis cualitativo de la información se enmarcó en el siguiente procedimiento: I) ordenamiento de conceptos y categorías extraídos de las fuentes de información (coincidencia de descriptores y año de publicación); II) agrupamiento de conceptos y categorías símiles; III) redacción de conceptos a partir de confluencias en los descriptores y IV) resultados y discusión. Para cumplir nuestro objetivo y aportar en la comprensión de nuestro objeto de estudio se realizó un proceso de redacción de conceptos mediante una construcción sintáctica, morfológica y pragmática de las confluencias teóricas encontradas en las fuentes de información.

\section{RESULTADOS}

Los registros obtenidos no superaron las 30 fuentes tras la combinación de las diferentes palabras clave y sus posibles sinónimos de búsqueda, siendo utilizadas únicamente como referencias en la fuente. La totalidad de fuentes encontradas que abordan la "transferencia de tecnología" concuerdan en que es un concepto complejo, difuso y en constante transformación con un sinnúmero de variables de análisis en dependencia de la perspectiva del autor y la contextualización de su obra; podemos resaltar algunos elementos teóricos con mayor prevalencia a la hora de conceptualizar el fenómeno. El proceso de transferencia de tecnología implica siempre el movimiento de "know-how", de conocimiento científico o de tecnología, de una organización a otra (ROESSNER, 2000; CASTRO, E. et al, 2008).

A partir de lo propuesto por Souza (1983) autores como Costa y Torkomian (2008) y Prysthon y Schmidt (2002), consideran que la transferencia de tecnología consiste en el desplazamiento de un conjunto de conocimientos y prácticas tecnológicas de una entidad para otra y que la transferencia de tecnología se produce cuando el receptor absorbe conocimiento conjunto que les permite innovar. En resumen, para que se realice la transferencia de tecnología deben existir los siguientes elementos: conocimiento científico, transferencia de información (saberes), un emisor (generador de conocimiento) y un receptor (el que se apropia del conocimiento).

No se encontraron coincidencias de los descriptores "mecanismos de transferencia" y "profesores de enseñanza media técnico agrícola" juntos en una misma fuente, se intentó ampliar el alcance de las variables a "profesores de 
enseñanza media", utilizando sinónimos de búsqueda "docentes - instructores tutores + educación media + bachillerato técnico" en los tres idiomas de búsqueda, sin coincidencias. Sin embargo, en cuanto a los descriptores con la variable aislada "mecanismos de transferencia de tecnología" distintos autores como López, S.; Mejía, J.C.; Schmal, R. (2006); Peña, M.; Ríos J. Aristizábal C. (2011); Costa y Torkomian (2008), Ginsparg, P. (1996), Nilsson, Rickne e Bengtsson (2010) y Martins Dos Santos D. (2012) concuerdan en que los mecanismos de transferencia más utilizados en la actualidad son las publicaciones científicas en medios especializados disponibles en internet e impresos, los spin-offs y patentes, además de estos que presentan mayor frecuencia en las fuentes, se destacan las oficinas de transferencia tecnológica de los centros de producción científica y otras organizaciones de intermediación.

La búsqueda conjunta de las variables "profesores de enseñanza media técnica agrícola" y "apropiación de saberes - actualización de conocimientos" no arrojó coincidencias en ninguna de las bases indexadas. Así mismo, se intentó la búsqueda con sus sinónimos sin éxito. Una búsqueda sencilla en Google académico arrojó solo tres coincidencias pero sin elementos referentes a "educación nivel técnico - agricultura". Se observa que desde la metodología empleada y las bases de datos elegidas para la investigación, no hay hallazgos significativos con relación a nuestro objeto de estudio: relación entre apropiación del conocimiento científico, docentes de colegios técnicos agrícolas y los mecanismos de transferencia de tecnología más utilizados por los docentes. En ese contexto, se distinguen dos corrientes de pensamiento claramente diferenciadas encontradas en las fuentes, ambas antagónicas, basadas en postulados completamente contrarios sobre la apropiación de saberes y del conocimiento científico. Por un lado están los que defienden el conocimiento como un bien público y por lo tanto de libre acceso para toda la población y para todas las organizaciones, por el otro lado están los que consideran que la única forma de que avance el progreso científico y tecnológico es a través de la protección jurídica de los inventores y de los inventos y las creaciones individuales y grupales. La apropiación del conocimiento gracias a la aparición de las nuevas tecnologías de la información y la comunicación permiten una concepción de la "apropiación social del conocimiento" (ECHEVERRI R. y FRANCO L., 2012; BARRIO C., 2008).

Otro hallazgo significativo es la noción de "formación continua", esta variable se encontró en todas las bases de datos consultadas con una frecuencia de aparición mayor que los demás descriptores de búsqueda, tal variable es motivo de investigaciones correlacionada con algunas otras variables pensamiento cientifico y mecanismos de trasferencia de tecnología e innovación mayormente. Esta categoría apareció en fuentes relacionada a "conocimiento científico", "pensamiento/hallazgos científicos e innovación tecnológica", "apropiación de saberes" y "mecanismos de trasferencia de información". Siendo una constante la descripción de la misma y la importancia para el desarrollo personal. El proceso de formación de los docentes no culmina con la formación básica, pues faltará no sólo la profundización de todas las problemáticas científicas y educativas, apenas abiertas durante los cursos de profesorado, sino fundamentalmente la reflexión sistemática sobre la práctica didáctica. Para todos los casos, la formación y actualización docente es concebida como un proceso continuo, que comienza con la formación básica y se prolonga más allá de ésta, constituyendo una carrera a lo largo de toda la vida donde prima la búsqueda de información científica para perfeccionar la actividad docente (GOUVEIA, 1992; PACCA, 1994; FURIÓ, C.J. 1994; PACCA, J. y VILLANI, A., 2000,). 
La búsqueda de los descriptores "transferencia de tecnología" y "transferencia de información" arrojó cinco fuentes, pudiéndose evidenciar que existen diferencias marcadas entre ambas. La primera implica, necesariamente, reciprocidad mientras que la segunda no siempre presenta esta característica. La transferencia de tecnología y la transferencia de información se basan, en principio, en los conceptos de tecnología de la información y la información científica. La tecnología de la información, es un conjunto de "saberes y prácticas" que son paquetes descargables a través de acuerdos entre las partes, que implican una concesión de "know-how" específicos para replicar procesos, productos y servicios. Así mismo, las fuentes consultadas concuerdan en que los procesos transferencia de tecnología e información hacia instituciones educativas o empresas del sector público o privado contribuyen a la creación de nuevas empresas y empleos en distintos sectores y estratos de la población (MIRANDA, A. y SIMEÃO, E., 2004; JOVA et al., 2006; LÓPEZ, S.; MEJÍA, J.C.; SCHMAL, R., 2006; LÓPEZ PEÑA, M.; RÍOS J. ARISTIZÁBAL C., 2011).

\section{CONSIDERACIONES FINALES}

Después de realizar la pesquisa y de revisar las coincidencias en las fuentes surgieron nuevas dudas, todas ellas direccionadas hacia la correlación de los descriptores con nuevos elementos encontrados en los artículos revisados. Como una primera conclusión que se desprende de la investigación está el hecho de que no existe una teoría definitiva o acabada en torno al proceso de transferencia de tecnología, conocimiento científico e innovación y es necesario analizar el fenómeno desde muchas otras perspectivas no encontradas en las bases de datos seleccionadas, como los determinantes sociales, históricos, políticos y económicos de los actores donde se realiza el proceso apropiación de saberes. Se abre una nueva posibilidad de estudios posteriores que ayuden a comprender mejor el fenómeno.

Uno de los resultados más relevantes es la distinción teórica entre la transferencia de tecnología y la transferencia de información; ambas parten de uno de los descriptores de la presente investigación, el conocimiento científico, pero tienen una connotación diferente, básicamente la primera se refiere a los productos, paquetes tecnológicos con herramientas (bienes de consumo) y la transferencia de información se refiere exclusivamente al contenido. Se observa en las fuentes de información que la transferencia de tecnología responde a intereses económicos ya que las ganancias de las transacciones realizadas financian las investigaciones y sustentan el mantenimiento de las mismas mientras que la transferencia de información no necesariamente se basa en una relación económica, podría ser de índole cognitiva, informativa, ética, política o social. En ambos procesos de trasferencia se incluyen saberes, tecnología, ciencia e innovación y las nuevas tecnologías de la información juegan un papel preponderante. La transferencia de tecnología y del conocimiento científico implican reciprocidad en una relación de intercambio de información como paquetes tecnológicos con herramientas para su estudio y desarrollo, mientras que la transferencia de información no siempre es recíproca, puede ser unidireccional y mediada por el uso de internet.

Sobre el proceso de transferencia de información (conocimiento científico) en docentes de Colegios Técnicos Agrícolas, no se encontraron referencias que brinden luces sobre esta relación, sin embargo; se puede hacer una generalización empírica a partir las coincidencias arrojadas por la metodología RAD sobre docentes de enseñanza media técnica general, en este caso, los docentes cuando se apropian del conocimiento lo hacen a partir de información científica, innovación, hallazgos, 
productos y servicios, implica en la totalidad de los casos el desplazamiento de conocimientos y resulta eficaz sólo cuando logra absorber el conocimiento que le permitirá innovar y mejorar procesos, siendo capaz de desarrollar nuevas tecnologías y más conocimiento a partir de lo aprendido; en decir, cuando consigue transformar, innovar y crear conocimiento científico.

Se hace evidente que en los últimos años se han incrementado las publicaciones científicas que abordan la variable "formación continua". Coinciden las fuentes en que la apropiación de saberes y actualización de conocimientos por parte de los docentes es un proceso de formación constante que no acaba con la obtención de un título académico, ya que existen conocimientos técnicos que están en constante cambio y el docente debe suplir estas necesidades educativas, pedagógicas e investigativas con información científica actualizada, en concordancia con la disciplina que imparte y los nuevos hallazgos científicos y tecnológicos. Se observó en este contexto la importancia de las nuevas tecnologías de la comunicación y las redes, en primera instancia por el uso que hacen de ella los docentes para actualizar conocimientos, destacándose los beneficios como inmediatez, bajo costo y accesibilidad y además, el incremento de su uso por parte de los centros de producción científica para divulgar resultados de investigaciones.

La bibliografía sugiere que los mecanismos de transferencia más utilizados en la actualidad son las publicaciones científicas en medios especializados, "spinoffs" académicos, licencias y patentes. Sin embargo, los docentes, de manera general actualizan sus conocimientos y acceden a la información gracias a publicaciones científicas en formato virtual, no necesariamente en bases indexadas. Estas publicaciones son de fácil acceso gracias al internet, trayendo consigo un nuevo tipo de relación entre el conocimiento científico y la apropiación de los mismos, en la actualidad es un proceso mucho más directo, accesible e inmediato, pero podría acarrear una serie de elementos no favorables como la falta de retroalimentación y el riesgo de encontrar información que no se corresponda con contenidos científicos creando sesgos y distorsión.

Se logró apreciar en las fuentes analizadas que el proceso de transferencia de tecnología se aborda desde una perspectiva empresarial, basada en el capital y orientada hacia el mercado. Las fuentes abordadas en esta investigación no contemplaron elementos como características socioeconómicas, históricas, políticas, culturales o personales dentro del esquema de reciprocidad que teóricamente implica la relación entre los centros de producción científica y los docentes / investigadores. Queda abierta la posibilidad de realizar pesquisas científicas que aborden el fenómeno de la trasferencia de conocimiento científico, la formación continua o los mecanismos de trasferencia desde alguna de las perspectivas antes mencionadas.

\section{BIBLIOGRAFÍA}

ALESSANDRINI, M., KLOSE, K.; PEPPER, M. University entrepreneurship in South Africa: developments in technology transfer practices. Innovation: Management, Policy, \& Practice. v. 15, 2013. Disponible en: https://www.tandfonline.com/doi/abs/10.5172/impp.2013.15.2.205. Acceso en: 17 dic. 2016.

ANDERSON, T., DAIM, T.; LAVOIE, F. Measuring the efficiency of university technology transfer. Technovation, 27(5), p. 306-318. 2007. Disponible en: https://www.researchgate.net/publication/223282455_Measuring_the_efficiency_of_u niversity_technology_transfer. Acceso en: 10 dic. 2016. 
BARBOSA, J. W.; BARBOSA, J. C.; RODRÍGUEZ, M. BARBOSA, J. et al Revisión y análisis documental para estado del arte: una propuesta metodológica desde el contexto de la sistematización de experiencias educativas. Investigación bibliotecológica. vol.27 no.61. 2013. Disponible en: http://www.scielo.org.mx/scielo.php?pid=S0187358X2013000300005\&script=sci_arttext. Acceso en: 05 de dic. 2016

BARRIO C. La apropiación social de la ciencia: nuevas formas. Revista Iberoamericana de Ciencia Tecnología y Sociedad. v.4 n.10, 2008. Disponible en: http://www.scielo.org.ar/scielo.php?pid=S185000132008000100014\&script=sci_arttext. Acceso en: 05 de nov. 2016

BOZEMAN, B. Technology transfer and public policy: A review of research and theory. Research Policy, 627-655. 2000. Disponible en: https://www.sciencedirect.com/science/article/pii/S0048733399000931. Acceso en: 17 dic. 2016.

BOZU, Z. El perfil de las competencias profesionales del profesorado de la ESO (Educación Secundaria Obligatoria). Departamento de Didáctica y Organización Educativa. Universitat de Barcelona, 2007. Disponible en: http://www.oei.es/docentes/articulos/perfil competencias profesionales profesorado _eso_bozu.pdf. Acceso en: 05 de dic. 2016.

CASTRO, E.; FERNÁNDEZ, I.; PÉREZ, M.; CRIADO, F. La transferencia de conocimientos desde las humanidades: posibilidades y características. ARBOR Ciencia, Pensamiento y Cultura. CLXXXIV. 619-636, 2008. Disponible en: http://arbor.revistas.csic.es/index.php/arbor/article/view/211/212. Acceso en: 05 de nov. 2016

COSTA, L.; TORKOMIAN, L. Um estudo exploratório sobre um novo tipo de empreendimento: os spin-offs acadêmicos. RAC - Revista de Administração Contemporânea, $2008 . \quad$ Disponible http://www.scielo.br/scielo.php?script=sci_abstract\&pid=S141565552008000200006\&lng=pt\&nrm=iso\&tIng=en. Acceso en: 17 dic. 2016.

COTEC. Nuevos mecanismos de transferencia de tecnología Debilidades y oportunidades del Sistema Español de Transferencia de Tecnología. Encuentros Empresariales. Fundación COTEC para la Innovación Tecnológica, 2002. Disponible en: http://informecotec.es/media/I09_New_Mec_Transf_Tec.pdf. Acceso en: 05 de nov. 2016

ECHEVERRI R.; Y FRANCO L. Apropiación del conocimiento: análisis de dos lógicas desde una perspectiva sistémica. Ponencia $X$ Congreso Latinoamericano de Dinámica de Sistemas, Anais... 2012.

FERRANDEZ, R.; SANCHEZ, L. Competencias docentes en secundaria. Análisis de perfiles de profesorado. RELIEVE: Revista Electrónica de Investigación y

Evaluación Educativa 20.1, 2014. Academic OneFile. Disponible en: https://ojs.uv.es/index.php/RELIEVE/article/view/3786. Acceso en: 15 de nov. 2016. 
FELDMAN, M., FELLER, I., BERCOVITZ, J.; BURTON, R. Equity and the technology transfer strategies of American research universities. Management. Science, vol. 48, pp. 105-121, 2002.

FLORES, G. Retos y problemáticas en la formación de docentes de educación básica un acercamiento a los procesos cognitivos. X Congreso Nacional de Investigación Educativa, 2009. Disponible en: http://www.comie.org.mx/congreso/memoriaelectronica/v10/pdf/area_tematica_15/po nencias/0931-F.pdf. Acceso en: 17 de dic. 2016.

FURIÓ, C.J. Tendencias actuales en la formación del profesorado de ciencias. Departamento de Didáctica de les Ciencias Experimentales y Sociales. Universidad de Valencia, España. Enseñanza de las ciencias, 188-199, 1994.

GINSPARG, P. Electronic publishing in science. Winners and losers in the global research village. Contribution for Conference held at UNESCO HQ, Paris, 19-23, Feb 1996, during session Scientist's View of Electronic Publishing and Issues Raised. Disponible en: http://www.cs.cornell.edu/ ginsparg/physics/blurb/pg96unesco.html. Acceso en: 15 de nov. 2016

GONZÁLEZ, M.; SÁNCHEZ, Y.; ALCAIDE, Y.; VÁZQUEZ, M.; ARTEAGA, B.; CECE, L. Comportamiento de las publicaciones científicas. Educación Médica Superior, Ciudad de La Habana, v. 29, n. 3, sep. 2015. Disponible en http://scielo.sld.cu/scielo.php?script=sci_arttext\&pid=S0864-

$21412015000300008 \&$ Ing=es\&nrm=iso. Acesso en: 12 dic. 2016.

GONZALEZ SUAREZ, E. Conocimiento científico e información científica. ACIMED, Ciudad de La Habana, v. 14, n. 6, dic. 2006. Disponible en http://scielo.sld.cu/scielo.php?script=sci_arttext\&pid=S1024-

$94352006000600003 \&$ lng=es\&nrm=iso. Acesso en: 12 dic. 2016.

GÓMEZ, J. M.; MIRA, I.; VERDÚ, A. J. Las spin-off académicas como vía de transferencia tecnológica. Economía industrial, ISSN 0422-2784, № 366, 61-72, 2007. Disponible en: https://dialnet.unirioja.es/servlet/articulo?codigo=2510925. Acceso en: 15 de nov. 2016

JOVA, S., OJEDA R.; PEÑA L. La transferencia de tecnología, la innovación tecnológica y su incidencia en el desarrollo local. Folletos Gerenciales 10.11 43+, 2006. Disponible en: Academic OneFile. Acceso en: 15 de nov. 2016

LÓPEZ, H. El proceso de transferencia de tecnología: caso UPDCE. Tesis para obtener el grado de Máster en política y gestión del cambio tecnológico. México, D F. 2010.

LÓPEZ, S.; MEJÍA, J.C.; SCHMAL, R. Un acercamiento al concepto de la transferencia de tecnología en las universidades y sus diferentes manifestaciones.

Panorama socioeconómico.Vol.24, No. 32, p.70-81, 2006.

MARCONI, M.A.; LAKATOS, E.M. Fundamentos de metodologia científica. 6.ed. São Paulo: Editorial Atlas, 2005. 
MARTINS DOS SANTOS D. Critérios de eficácia do modelo de Bozeman e a transferência de tecnologia a partir de conhecimento gerado em universidade pública: estudo de casos múltiplos. (Mestrado em Administração) Universidade Federal do Paraná, 2012.

MIRANDA, A.; SIMEÃO, E. Transferência de informação e transferência de tecnologia no modelo de Comunicação Extensiva: a babel.com. Información, Cultura y Sociedad vol 10, .2004.

MIRANDA, C.; RIVERA P. Formación permanente de profesores: ¿quién es el formador de formadores? Estudios Pedagógicos, 35.1 155+, 2009. Disponible en: https://scielo.conicyt.cl/scielo.php?script=sci_abstract\&pid=S071807052009000100009\&lng=es\&nrm=iso\&tlng=en. Acceso en: 15 de nov. 2016

MORAES, M. D. et al. Estratégias de difusão do conhecimento da pesquisa agropecuária da universidade do estado de Mato Grosso. Extensão Rural, Santa Maria, n. 22, p. 29-66, 2011. Disponível en: https://periodicos.ufsm.br/extensaorural/article/view/5574/3295. Acesso en: 02 ene. 2017.

OPPENHEIM, C.; GREENHALGH, C.; ROWLAND, F. The future of scholarly jornal publishing. Journal of Documentation, 56 (4), p.361-398. 2000

PACCA, J.; VILLANI, A. La competencia dialógica del profesor de ciencias en Brasil. Instituto de Física. USP, São Paulo. Enseñanza de las Ciencias, p. 95-104, 2000.

PEÑA, M., RÍOS J.; ARISTIZÁBAL, C. Los procesos de transferencia de conocimiento en algunas universidades colombianas. Revista Escenarios, n. 11, p 34-48, 2011.

PÉREZ, M.; NÚÑEZ, A. Caracterización del proceso de la Transferencia de Tecnología en Instituciones de Educación Superior Mexicanas, 2013. Disponible en: http://www.altec2013.org/programme_pdf/1047.pdf. Acceso en: 25 de nov. 2016

PRYSTHON, C.; SCHMIDT, S. A experiência do Leaal/UFPE na produção e transferência de tecnologia. Ciência da Informação, V 31 N1, 84-90, 2002. Disponible en: http://www.scielo.br/scielo.php?script=sci_abstract\&pid=S0100$19652002000100009 \& \operatorname{lng}=e s \& n r m=i s o \& t \operatorname{lng}=e n$. Acceso en: 25 de nov. 2016

RUSSELL, J.M. Tecnologias electrônicas de comunicaçâo: bônus ou ônus para os cientistas dos países em desenvolvimento? Comunicaçâo Científica, Estudos Avançados em Ciência da Informaçâo V. 1. p. 35-49. 2000

TEJADA, J. Competencias Docentes. Revista Profesorado, vol. 13, № 2, 2009. Disponible en: http://digibug.ugr.es/bitstream/10481/7373/1/rev132COL2.pdf. Acceso en: 15 de nov. 2016 